Atıf/Citation: M. H. PEKALP, H. AYDOĞDU, "Sabit Bekleme Zamanlı Tip II Sayaç Sürecinde Ortalama Değer ve Varyans Fonksiyonlarının Parametrik Tahmini”, Süleyman Demirel Üniversitesi Fen Edebiyat Fakültesi Fen Dergisi, 14, 28-38, 2019.

\title{
Sabit Bekleme Zamanlı Tip II Sayaç Sürecinde Ortalama Değer ve Varyans Fonksiyonlarının Parametrik Tahmini
}

\author{
Mustafa Hilmi PEKALP ${ }^{* 1}$, Halil AYDOĞDU ${ }^{1}$ \\ ${ }^{1}$ Ankara Üniversitesi, Fen Fakültesi, İstatistik Bölümü, 06100, Ankara, Türkiye \\ *yazışllan yazar e-posta: mpekalp@ankara.edu.tr
}

(Alinış / Received: 26.06.2018, Kabul / Accepted: 20.12.2018, Yayımlanma / Published: 31.05.2019)

Özet: Bu çalışmada gecikmeli bir yenileme süreci olarak oluşturulan sabit bekleme zamanlı tip II sayaç sürecinin ortalama değer ve varyans fonksiyonları analitik olarak elde edilmektedir. Gecikmeli yenileme süreçleri ile ilgili uygulamalarda genellikle dağılım fonksiyonları şekilsel olarak bilinirken bazı parametreleri bilinmez. $\mathrm{Bu}$ durumda ortalama değer ve varyans fonksiyonları veriden tahmin edilmek zorundadır. Bu çalıșmada tip II sayaç sürecinin ortalama değer ve varyans fonksiyonları analitik olarak elde edildikten sonra, analitik ifadelerine bağlı biçimde, parametrik olarak bu fonksiyonların değerlerinin tahmin edilmesi üzerinde durulmakta ve bazı tahmin ediciler önerilmektedir. Bu tahmin edicilerin performansları küçük örneklem hacimleri için yan, hata kareler ortalaması kriterlerine göre bir simülasyon çalışması ile değerlendirilmektedir.

Anahtar kelimeler: Yenileme süreci, Gecikmeli yenileme süreci, Tip II sayaç süreci, Ortalama değer fonksiyonu, Varyans fonksiyonu, Parametrik tahmin.

\section{Parametric Estimation of the Mean Value and Variance Functions in Type II Counter Process with Constant Locking Time}

\begin{abstract}
In this study, the mean value and variance functions of type II counter process constituted as a delayed renewal process are obtained analytically. In the applications of the delayed renewal process, generally, the functional forms of the distributions are known but some parameters of the distributions are unknown. In those cases, the mean value and the variance functions must be estimated from the data. In this study, after obtaining the mean value and variance functions of type II counter process analytically, depending on the analytical expressions, we deal with the problem of estimating these functions and some estimators are proposed. Performance of the estimators is evaluated for small sample sizes by a simulation study according to bias and mean square error criteria.
\end{abstract}

Keywords: Renewal process, Delayed renewal process, Type II counter process, Mean value function, Variance functions, Parametric estimation.

\section{Giriş}

Yenileme süreçleri envanter, kuyruk, risk, güvenirlik teorisi ve uygulamalı istatistiğin birçok alanında kullanılan önemli bir sayma süreci modelidir. Bu sayma süreci ile ilgili bazı tanımlamalar ve kavramlar aşağıdaki gibi verilir.

Tanım: $\left\{X_{k}, k=1,2, \ldots\right\}$ pozitif değerli, bağımsız ve aynı $F$ dağılım fonksiyonuna sahip rasgele değişkenlerin bir dizisi olsun. $X_{1}$ rasgele değişkeni ilk olay (yenileme) 
gerçekleşinceye kadar geçen zaman olmak üzere $X_{k}$ rasgele değişkeni $(k-1)$. ve $k$. olaylar (yenilemeler) arasında geçen zamanı temsil etsin. $S_{0}=0, S_{n}=X_{1}+X_{2}+\cdots+$ $X_{n}, n=1,2, \ldots$ ve $N(t)=\operatorname{maks}\left\{n: S_{n} \leq t\right\}, t$ zamanına kadar, yani $(0, t]$ zaman aralığında yapılan yenilemelerin sayısı olmak üzere $\{N(t), t \geq 0\}$ stokastik sürecine alışılmış anlamda bir yenileme süreci denir.

$\left\{X_{k}, k=1,2, \ldots\right\}$ pozitif değerli, bağımsız ve $1 / \lambda$ parametresi ile üstel dağılıma sahip rasgele değişkenler olduğunda bu dizi üzerine kurulu $\{N(t), t \geq 0\}$ yenileme süreci $\lambda$ oranlı homojen bir Poisson süreci olur.

$\{N(t), t \geq 0\}$ alışılmış anlamda bir yenileme süreci olmak üzere $M(t)=E(N(t)), t \geq$ 0 ile verilen $M$ fonksiyonuna yenileme sürecinin ortalama değer fonksiyonu denir. $\mathrm{Bu}$ fonksiyon aynı zamanda yenileme fonksiyonu olarak da adlandırılır. $M$ yenileme fonksiyonu için aşağıdaki eşitlik geçerlidir.

$M(t)=\sum_{k=1}^{\infty} F^{k *}(t), t \geq 0$

Burada $F^{k *}, F^{\prime}$ nin kendisi ile $k$ kez Stieltjes konvolüsyonudur. Ayrıca $M$ yenileme fonksiyonu yenileme denklemi olarak bilinen aşağıdaki integral denklemi sağlar.

$M(t)=F(t)+\int_{0}^{t} M(t-x) d F(x), t \geq 0$

Yukarıdaki yenileme denklemine Laplace-Stieltjes dönüşümünün uygulanmasıyla

$M_{L S}(t)=\frac{F_{L S}(t)}{1-F_{L S}(t)}, t>0$

bulunur. Burada $M_{L S}$ ve $F_{L S}$ fonksiyonları sirasiyla $M_{L S}(t)=\int_{0}^{\infty} e^{-t x} d M(x), t \geq 0$ ve $F_{L S}(t)=\int_{0}^{\infty} e^{-t x} d F(x), t \geq 0$ ile tanımlanan $M$ ve $F$ fonksiyonlarının LaplaceStieltjes dönüşümleridir [5,6].

$\{N(t), t \geq 0\}$ alışılmış anlamda bir yenileme süreci olmak üzere $V(t)=E\left(N^{2}(t)\right)-$ $E(N(t))^{2}, t \geq 0$ ile verilen $V$ fonksiyonuna yenileme sürecinin varyans fonksiyonu denir. $V$ varyans fonksiyonu konvolüsyon serilerine bağlı olarak aşağıdaki gibi elde edilir.

$V(t)=2 \sum_{k=1}^{\infty} k F^{k *}(t)-\sum_{k=1}^{\infty} F^{k *}(t)\left(1+\sum_{k=1}^{\infty} F^{k *}(t)\right), t \geq 0$

Ayrıca $V$ varyans fonksiyonu için

$V(t)=M(t)(1-M(t))+2 M * M(t), t \geq 0$

dır [5,7]. Burada belirtelim ki her $t \geq 0$ için $M(t)$ ve $V(t)$ sonludur.

Tanım: Pozitif değerli ve bağımsız $\left\{X_{k}, k=1,2, \ldots\right\}$ rasgele değişkenleri için $X_{2}, X_{3}, \ldots$ rasgele değişkenleri $F$ dağılım fonksiyonu ile aynı dağılıma sahip iken, $X_{1}$ rasgele değişkeni $F$ 'den farklı bir $G$ dağılım fonksiyonuna sahip ise $\left\{X_{k}, k=1,2, \ldots\right\}$ rasgele değişken dizisi üzerine kurulu olarak $N_{d}(t)=\operatorname{maks}\left\{n: S_{n} \leq t\right\}$ ile verilen $\left\{N_{d}(t), t \geq\right.$ $0\}$ sayma sürecine bir gecikmeli yenileme süreci denir, burada $S_{n}=X_{1}+X_{2}+\cdots+X_{n}$, $n=1,2, \ldots$ 
$\left\{N_{d}(t), t \geq 0\right\}$ bir gecikmeli yenileme süreci olmak üzere $M_{d}(t)=E\left(N_{d}(t)\right), t \geq 0$ ile tanımlanan $M_{d}$ fonksiyonuna gecikmeli yenileme sürecinin ortalama değer fonksiyonu denir. $M_{d}$ ortalama değer fonksiyonu için aşağıdaki eşitlik geçerlidir.

$M_{d}(t)=\sum_{k=1}^{\infty} G * F^{(k-1) *}(t), t \geq 0$

burada $F^{0 *}(t)=\left\{\begin{array}{l}0, t<0 \\ 1, t \geq 0\end{array}\right.$. Ayrıca $M_{d}$ gecikmeli yenileme fonksiyonunun

$M_{d}(t)=G(t)+\int_{0}^{t} M_{d}(t-x) d F(x), t \geq 0$

integral denklemini sağladığı bilinen bir sonuçtur. Bu integral denkleme LaplaceStieltjes dönüşümünün uygulanması ile

$\left(M_{d}\right)_{L S}(t)=\frac{G_{L S}(t)}{1-F_{L S}(t)}, t>0$

bulunur $[5,6]$.

$\left\{N_{d}(t), t \geq 0\right\}$ bir gecikmeli yenileme süreci olmak üzere $V_{d}(t)=E\left(N_{d}^{2}(t)\right)$ $E\left(N_{d}(t)\right)^{2}, t \geq 0$ ile verilen $V_{d}$ fonksiyonuna gecikmeli yenileme sürecinin varyans fonksiyonu denir. $V_{d}$ varyans fonksiyonu aşağıda verilen integral denklemi sağlar.

$V_{d}(t)=M_{d}(t)\left(1-M_{d}(t)\right)+2 M_{d} * M(t), t \geq 0$

(9) eşitliğinde $M$ ve $M_{d}$ fonksiyonlarının sırasıyla (1) ve (6) konvolüsyon serilerine bağlı ifadeleri göz önüne alındığında

$V_{d}(t)=\sum_{k=1}^{\infty} G * F^{(k-1) *}(t)\left(1-\sum_{k=1}^{\infty} G * F^{(k-1) *}(t)\right)+2 \sum_{k=1}^{\infty} k G * F^{k *}(t)$

olarak bulunur [5,7].

Burada not edelim ki yukarıda verilen $M_{d}(t)$ ve $V_{d}(t)$ fonksiyonları her $t \geq 0$ için sonludur.

Yenileme sürecinin önemli uygulama alanlarından biri de matematik ve fizik literatüründe şıkça kullanılan ve belirli bir kaynaktan yayılan parçacıkları sayan sayaçlardır. Sayaçlar, bir kaynaktan yayılan parçacıkları tespit eder ve kayıt altına alır. Ancak yayılan her parçacık sayaç tarafından tespit edilemeyebilir. Bir parçacığın sayaç tarafından kaydedilmesinden sonra bu parçacık sayaç tarafından çözümlenme aşamasına gireceğinden belli bir süre kendini kapatmak zorunda kalır. Bu nedenle bu sürede sayaca ulaşan parçacıklar kayıt altına alınamaz. Parçacıkların kayıt altına alınmadıkları bu zamana bekleme zamanı denir. Sayaca ilk ulaşan parçacık hiç beklemeden kaydedilir. Bundan sonraki aşamada ise sayacın bekleme zamanını tamamlamasıyla sayaca ulaşan parçacık kaydedileceğinden, ilk kaydedilme zamanı dışındaki diğer kaydedilme zamanlarının aynı dağılımlı olduğu, ancak ilk kaydedilme zamanından farklı bir dağılıma sahip olacakları açıktır. Böylelikle, parçacıkların gelişleri bir yenileme sürecine göre gerçekleştiğinde kaydedilen parçacıklar arası geçen zaman dizisi bir gecikmeli yenileme süreci oluşturur. Sayaçların bekleme zamanının belirlenmesi problemi ve bazı uygulama alanlarına ilişkin olarak $[8,9,10]$ kaynakları incelenebilir. $\mathrm{Bu}$ çalışmada belirli bir radyoaktif kaynaktan yayılan parçacıkların $\lambda$ oranı ile bir Poisson sürecine göre yayıldıkları, sayacın parçacığı kaydetmesi ile belirli ve sabit bir $L$ 
süre kapalı kaldığ 1 durum gözönüne alınır. Bu durumda kaydedilen parçacıklar arası geçen zamanlar tip II sayaç süreci olarak adlandırılan bir gecikmeli yenileme süreci oluşturur [5]. $\mathrm{Bu}$ sürecin ortalama değer fonksiyonunun analitik ifadesi literatürde verilmiştir [5]. Fakat şu ana kadar bu sürecin varyans fonksiyonu için herhangi bir analitik ifade elde edilmemiştir. Bu çalışmada $M_{d}(t)$ 'nin yanı sıra $V_{d}(t)$ için analitik bir ifade elde edilmektedir. Bu analitik ifadelere bağlı olarak bu fonksiyonların değerlerinin parametrik tahminleri bilinmeyen $\lambda$ oran parametresinin tahmini yardımıyla elde edilir. Ayrıca bu tahmin edicilerin tutarlı oldukları gösterilmektedir. Son olarak da bu tahmin edicilerin küçük örneklem özellikleri bir simülasyon çalışması ile hata kareler ortalaması ve yan kriterlerine göre değerlendirilmektedir.

\section{Tip II Sayaç Süreci için $M_{d}(t)$ ve $V_{d}(t)$ Fonksiyonlarının Analitik Hesabı}

Belli bir radyoaktif madde $\lambda$ oranlı bir $\left\{N_{1}(t), t \geq 0\right\}$ Poisson sürecine göre parçacıklar yaymaktadır. Radyoaktif maddeden yayılan parçacıkları tespit eden ve bu parçacıkları kaydeden bir sayacı göz önüne alalım. Bir parçacığın sayaç tarafından kaydedilmesi ile birlikte sayaç parçacığı analiz etmekte ve bu nedenle belirli sabit bir $L$ süre kapalı kalmaktadır. Parçacığın analizi sırasında sayaç kapalı olacağından sayaca ulaşan parçacıklar kaydedilmeyecektir. Sayaca ulaşan bir parçacı̆̆ın kaydedilmesi için gerek ve yeter şart sayacın kapalı kaldığı L süre boyunca hiçbir parçacığın gelmemesi olacağından bir parçacığın sayaç tarafından kaydedilmesi olasılı̆̆ı $p=e^{-\lambda L}$ olur. $Y_{1}, Y_{2}, \ldots$ rasgele değişkenleri kaydedilen parçacıklar arasında geçen zaman olarak tanımlandığında, bu rasgele değişkenler üzerine kurulu sayma süreci $\left\{N_{2}(t), t \geq 0\right\}$ olmak üzere $N_{2}(t),(0, t]$ aralığında sayaç tarafından kaydedilen parçacık sayısı olacaktır. $Y_{1}, Y_{2}, \ldots$ rasgele değişkenleri bağımsız olup, $Y_{1}$ rasgele değişkeni $\lambda$ parametreli $\left(1 / \lambda\right.$ ortalamalı) üstel dağılıma sahip iken, $Y_{2}, Y_{3}, \ldots$ rasgele değişkenleri aynı dağılımlı fakat $Y_{1}$ rasgele değişkeninin dağılımından farklıdırlar. Böylelikle $\left\{N_{2}(t), t \geq 0\right\}$ bir gecikmeli yenileme sürecidir. Bu sürecin ortalama değer ve varyans fonksiyonları sırasıyla (8) ve (9) ifadelerinden aşağıdaki gibi elde edilir.

$Y$, bağımsız ve aynı dağılımlı $Y_{2}, Y_{3}, \ldots$ rasgele değişkenlerinin bir temsilcisi olsun. $Y$, ilk parçacık kaydedildikten sonra herhangi kaydedilen iki parçacık arasındaki zamanı ifade eder. İlk olarak $Y$ rasgele değişkeninin dağılımını Laplace-Stieltjes dönüşümü yardımıyla bulalım. $Y$ sürede sayaç tarafından kaydedilmeyen parçacıkların sayısı $W$ olsun. Sayaca gelen parçacığın kaydedilmesi olasılığı $p=e^{-\lambda L}$ olduğundan $W$ rasgele değişkeninin olasılık fonksiyonu

$$
P(W=w)=e^{-\lambda L}\left(1-e^{-\lambda L}\right)^{w}, w=0,1, \ldots
$$

olur. $Y$ süresi boyunca, ilk kaydedilmiş parçacıktan sonra birinci kaydedilmemiş parçacık gelene kadar geçen zaman $U_{1}$ olmak üzere $i=2,3, \ldots$ için $U_{i},(i-1)$. kaydedilmemiş parçacık ile $i$. kaydedilmemiş parçacık arasında geçen zamanı ifade etsin. $U_{1}, U_{2}, \ldots$ rasgele değişkenlerinin bağımsız ve aynı dağılımlı olduğu açıktır. $V$ rasgele değişkeni son kaydedilmemiş parçacık ile kaydedilmiş parçacık arasında geçen zamanı göstersin. Varışlar bir Poisson sürecine göre gerçekleştiğinden $V, U_{1}, U_{2}, \ldots$ rasgele değişkenleri bağımsızdır. Böylece bu değişkenler yardımıyla $Y$ rasgele değişkeni

$Y=\sum_{i=1}^{W} U_{i}+V$

biçiminde ifade edilir. Bu durum görsel olarak tip II sürecinin bir gerçeklenişi ile aşağıdaki şekilde açıklanır. Aşağıda verilen şekilde $Y_{1} \sim$ Üstel $(\lambda)$ olmak üzere $i=$ $2,3, \ldots$ için $Y_{i} \stackrel{d}{=} Y^{\prime}$ dir. 


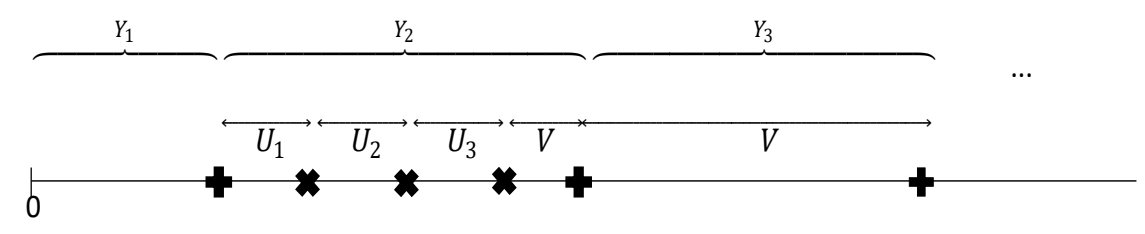

$\underbrace{W=3}_{\text {: Kaydedilen parçacık }} \underbrace{\frac{\boldsymbol{x}: \text { Kaydedilmeyen parçacık }}{W=0}}$

Şekil 1. Tip II sayaç sürecinin bir gerçeklenişi

Bağımsız ve aynı dağılımlı $U_{1}, U_{2}, \ldots$ rasgele değişkenleri bir $U$ rasgele değişkeni ile temsil edilsin. $X$ sayaca gelen iki parçacık arasında geçen zamanı gösteren, $\lambda$ parametresi ile üstel dağılımlı bir rasgele değişken olmak üzere $U=X \mid X<L$ ve $V=$ $X \mid X>L$ dir. $U$ ve $V$ rasgele değişkenlerinin dağılım fonksiyonları sırasıyla

$F_{U}(u)=P(X \leq u \mid X<L)= \begin{cases}\frac{1-e^{-\lambda u}}{1-e^{-\lambda L}}, & u \leq L \\ 1 & , u>L\end{cases}$

ve

$F_{V}(v)=P(X \leq v \mid X>L)=1-e^{-\lambda(v-L)}, v>L$

olur. $U$ ve $V$ rasgele değişkenlerinin dağılımlarının Laplace-Stieltjes dönüşümleri sirasiyla

$\phi_{U}(t)=\frac{\lambda}{1-e^{-\lambda L}} \frac{1}{t+\lambda}\left(1-e^{-L(t+\lambda)}\right)$

ve

$\phi_{V}(t)=\frac{\lambda e^{-L t}}{t+\lambda}$

biçiminde bulunur. $Y$ 'nin dağglımının Laplace-Stieltjes dönüşümü $W$ rasgele değişkeni üzerinden koşullandırma ile

$\phi_{Y}(t)=E\left(e^{-t Y}\right)=\sum_{w=0}^{\infty} E\left(e^{-t Y} \mid W=w\right) P(W=w)=\left\{1+\frac{t}{\lambda} e^{L(t+\lambda)}\right\}^{-1}$

dir. Bu durumda $\left\{N_{2}(t), t \geq 0\right\}$ gecikmeli yenileme sürecinin $M_{d}$ ortalama değer fonksiyonunun Laplace-Stieltjes dönüşümü (8) denkleminde $\phi_{X}(t)=\frac{\lambda}{\lambda+t}, t>-\lambda$ ve (11) ifadesinin kullanılmasıyla

$\phi_{M_{d}}(t)=\frac{\phi_{X}(t)}{1-\phi_{Y}(t)}=\frac{\lambda}{t(t+\lambda)}\left(t+\lambda e^{-L(t+\lambda)}\right), t>0$

bulunur. $\mathrm{Bu}$ ifadenin ters Laplace-Stieltjes dönüşümünün alınmasıyla $M_{d}$ ortalama değer fonksiyonu

$M_{d}(\mathrm{t})= \begin{cases}1-e^{-\lambda t} & , 0<t \leq L \\ 1-e^{-\lambda L}+\lambda(t-L) e^{-\lambda L}, & t \geq L\end{cases}$ 
olarak elde edilir.

Şimdi (9) ifadesini göz önüne alarak $\left\{N_{2}(t), t \geq 0\right\}$ gecikmeli yenileme sürecinin $V_{d}$ varyans fonksiyonunu analitik olarak elde edelim. Bunun için ilk olarak $Y$ rasgele değişkeninin dağılımına dayalı alışılmış anlamdaki yenileme sürecinin $M$ yenileme fonksiyonunu bulalım. (3) ifadesinin kullanılmasıyla

$\phi_{M}(t)=\frac{\phi_{Y}(t)}{1-\phi_{Y}(t)}=\frac{\lambda}{t} e^{-L(t+\lambda)}, t>0$

olup bu fonksiyonun ters Laplace-Stieltjes dönüşümünün alınmasıyla $M$ yenileme fonksiyonu

$M(t)= \begin{cases}0 & , 0<t \leq L \\ \lambda(t-L) e^{-\lambda L}, & t \geq L\end{cases}$

olarak bulunur. Bu durumda $M_{d} * M(t)$ konvolüsyonu hesaplamalar sonucunda

$M_{d} * M(t)= \begin{cases}0 & , 0<t \leq L \\ (t-L) \lambda e^{-\lambda L}+e^{-\lambda t}-e^{-\lambda L} & , L<t \leq 2 L \\ \left(\lambda L+e^{-\lambda L}-1\right) e^{-\lambda L}+(t-2 L) & \\ \times\left[\lambda e^{-\lambda(t+L)}-e^{-2 \lambda L}\left(\lambda e^{-\lambda L}+\lambda^{2} L-\frac{\lambda^{2} t}{2}\right)\right], t>2 L\end{cases}$

biçiminde elde edilir. Böylece $\left\{N_{2}(t), t \geq 0\right\}$ gecikmeli yenileme sürecinin $V_{d}$ varyans fonksiyonu (9) denkleminde (12) ve (13) ifadelerinin kullanılmasıyla

$V_{d}(\mathrm{t})= \begin{cases}1-e^{-\lambda L} & , 0<t \leq L \\ {\left[e^{-\lambda t}-\lambda e^{-\lambda L}(t-L)\right]\left[1-e^{-\lambda t}+\lambda e^{-\lambda L}(t-L)\right]} & \\ +2\left[(t-L) \lambda e^{-\lambda L}+e^{-\lambda t}-e^{-\lambda L}\right] & , L<t \leq 2 L \\ {\left[e^{-\lambda t}-\lambda e^{-\lambda L}(t-L)\right]\left[1-e^{-\lambda t}+\lambda e^{-\lambda L}(t-L)\right]+2\left[\left(\lambda L+e^{-\lambda L}-1\right) e^{-\lambda L}\right.} & \\ \left.+(t-2 L)\left\{\lambda e^{-\lambda(t+L)}-e^{-2 \lambda L}\left(\lambda e^{-\lambda L}+\lambda^{2} L-\frac{\lambda^{2} t}{2}\right)\right\}\right], t>2 L\end{cases}$

biçiminde bulunur.

\section{3. $M_{d}(t)$ ve $V_{d}(t)$ Fonksiyonları için Parametrik Tahmin Ediciler}

Alışılmış anlamdaki bir yenileme sürecinin yenileme ve varyans fonksiyonlarının tahmin problemi $[1,2,3]$ tarafından ele alınmıştır. Çalışmanın bu bölümünde gecikmeli yenileme süreci üzerine kurulu olan tip II sayaç sürecinin ortalama değer ve varyans fonksiyonlarının parametrik tahmini üzerinde durulur.

Genelde bir $\left\{N_{d}(t), t \geq 0\right\}$ gecikmeli yenileme sürecinde, sürecin ortalama değer ve varyans fonksiyonlarının parametrik tahmini aşağıdaki gibi elde edilir. Kabul edelim ki $G$ ve $F$ dağılım fonksiyonları şekilsel olarak bilinirken bazı parametreleri bilinmesin. $\theta_{1}, \theta_{2}, \ldots, \theta_{r}, G$ dağılımının ve $\gamma_{1}, \gamma_{2}, \ldots, \gamma_{s}, F$ dağılımının bilinmeyen parametreleri olmak üzere $G=G\left(\theta_{1}, \theta_{2}, \ldots, \theta_{r}\right)$ ve $F=F\left(\gamma_{1}, \gamma_{2}, \ldots, \gamma_{s}\right)$ yazalım. Kabul edelim ki, $Y_{1}, Y_{2}, \ldots, Y_{m}, G$ dağılımından $m$ birimlik rasgele bir örneklem ve $X_{1}, X_{2}, \ldots, X_{n}, F$ dağılımından $n$ birimlik rasgele bir örneklem olsun. $Y_{1}, Y_{2}, \ldots, Y_{m}$ örneklemine dayalı olarak elde edilen $\hat{\theta}_{1}, \hat{\theta}_{2}, \ldots, \hat{\theta}_{r}$ tahmin edicileri sirasiyla $\theta_{1}, \theta_{2}, \ldots, \theta_{r}$ parametrelerinin ve $X_{1}, X_{2}, \ldots, X_{n}$ örneklemine dayalı olarak elde edilen $\hat{\gamma}_{1}, \hat{\gamma}_{2}, \ldots, \hat{\gamma}_{s}$ tahmin edicileri sirasiyla $\gamma_{1}, \gamma_{2}, \ldots, \gamma_{s}$ parametrelerinin tahmin edicileri olmak üzere $G$ ve $F$ 'nin doğal 
tahmin edicileri sirasiyla $\widehat{G}_{m}=G\left(\hat{\theta}_{1}, \hat{\theta}_{2}, \ldots, \hat{\theta}_{r}\right)$ ve $\hat{F}_{n}=F\left(\hat{\gamma}_{1}, \hat{\gamma}_{2}, \ldots, \hat{\gamma}_{s}\right)$ ile verilir. $\hat{F}_{n}^{k *}$, $\widehat{F}_{n}$ 'nin k.ncı konvolüsyonu olmak üzere $M_{d}(t)$ ve $V_{d}(t)$ fonksiyonlarının sirasıyla (6) ve (10) ifadelerinde $G$ ve $F$ 'nin yerine tahmin edicileri olan $\hat{G}_{m}$ ve $\hat{F}_{n}$ alınmasiyla her sabit $t \geq 0$ için $M_{d}(t)$ ve $V_{d}(t)$ fonksiyonlarının parametrik tahmin edicileri

$\widehat{M}_{d}(t)=\sum_{k=1}^{\infty} \widehat{G}_{m} * \widehat{F}_{n}^{(k-1) *}(t), t \geq 0$

ve

$$
\begin{aligned}
\widehat{V}_{d}(t)=\sum_{k=1}^{\infty} \widehat{G}_{m} * \widehat{F}_{n}^{(k-1) *}(t)\left(1-\sum_{k=1}^{\infty} \widehat{G}_{m} * \widehat{F}_{n}^{(k-1) *}(t)\right) \\
+2 \sum_{k=1}^{\infty} k \widehat{G}_{m} * \widehat{F}_{n}^{(k-1) *}(t)
\end{aligned}
$$

ile tanımlanır.

$\widehat{M}_{d}(t)$ ve $\widehat{V}_{d}(t)$ tahmin edicilerine ilişkin asimptotik sonuçların elde edilebilmesi için $m$ 'nin $n$ 'ye bağl1, yani $m=m(n)$ biçiminde olduğu ve $n \rightarrow \infty$ iken $m \rightarrow \infty$ olduğu varsayılir.

Şimdi $\left\{N_{d}(t), t \geq 0\right\}$ tip II sayaç süreci olsun. Burada $G, \lambda$ parametresi ile üstel dağılım ve $F$, Laplace-Stieltjes dönüşümü (11) ile verilen dağılım fonksiyonudur. Bu modelde bilinmeyen tek parametre $\lambda$ 'dır. Bu durumda $\lambda$ 'nın süreçten gelen bir veri kümesine bağlı olarak tahmin edilmesiyle (12) ve (14) ifadelerinden her sabit $t \geq 0$ ve bilinen $L$ sabiti için $M_{d}(t)$ ve $V_{d}(t)$ fonksiyonlarının tahmin edicilerine ulaşılır: $\hat{\lambda}, \lambda$ 'nın bir tahmin edicisi olsun. (12) ve (14) ifadelerinde $\lambda$ yerine $\hat{\lambda}$ alınmasiyla her sabit $t \geq 0$ için $M_{d}(t)$ ve $V_{d}(t)$ 'nin tahmin edicileri sırasıyla aşağıdaki gibi elde edilir.

$\widehat{M}_{d}(t)= \begin{cases}1-e^{-\hat{\lambda} t} & , 0<t \leq L \\ 1-e^{-\widehat{\lambda} L}+\hat{\lambda}(t-L) e^{-\widehat{\lambda} L}, t \geq L\end{cases}$

ve

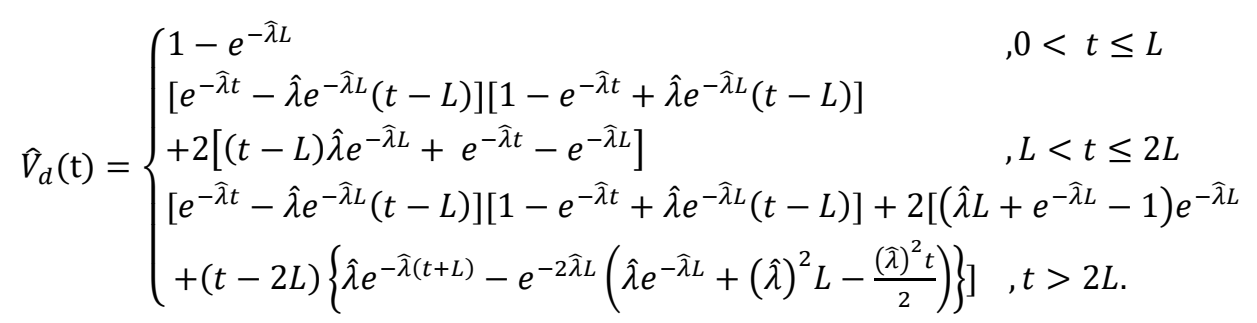

$\hat{\lambda}, \lambda$ parametresi için tutarlı bir tahmin edici olduğunda, sürekli dönüşüm teoreminden her sabit $t \geq 0$ için $\widehat{M}_{d}(t)$ ve $\widehat{V}_{d}(t)$ tahmin edicileri de $M_{d}(t)$ ve $V_{d}(t)$ için tutarlı tahmin ediciler olurlar.

(17) ve (18) ifadeleri göz önüne alındığında tip II sayaç sürecinin ortalama değer ve varyans fonksiyonlarının tahmin probleminin, $\lambda$ parametresinin tahmin problemine indirgendiği açıktır. Şimdi $\lambda$ parametresinin tahmin problemi için aşağıda verilen iki farklı örnekleme planını göz önüne alalım.

Örnekleme Planı 1: Tip II sayaç sürecini ilk olayı gerçekleşinceye kadar bağımsız olarak $m$-kez gözlemleyelim. Bu durumda $Y_{1}^{1}, Y_{1}^{2}, \ldots, Y_{1}^{m}, G$ dağılım fonksiyonundan 
gelen ve hacmi $m$ olan bir örneklem olacaktır. Burada $G$ dağılım fonksiyonunun $\lambda$ parametresi ile üstel dağılıma sahip olduğu açıktır. Bu örnekleme planına göre $\lambda$ parametresinin en çok olabilirlik tahmin edicisi $\hat{\lambda}=\frac{1}{m} \sum_{i=1}^{m} Y_{1}^{i}$ olur. Böylece her sabit $t \geq 0$ için, (17) ve (18) ifadeleri yardımıyla $M_{d}(t)$ ve $V_{d}(t)$ 'nin güçlü tutarlı tahmin edicilerine ulaşıllır.

Pratikte bir sürecin $m$ kez gözlemlenmesi kullanışlı bir gözlem yöntemi değildir. $\lambda, G$ ve $F$ dağılım fonksiyonlarının ortak parametresi olduğundan, bu parametre sürecin $n$ adımlık bir tek gerçekleşine karşılık gelen bir veri kümesine dayalı olarak aşağıdaki gibi tahmin edilebilir.

Örnekleme Planı 2: Tip II sayaç sürecini ardış1k $n$ tane olay gerçekleşinceye kadar gözlemleyelim. $\mathrm{Bu}$ durumda karşımıza çıkacak olan $\left\{Y_{1}, Y_{2}, \ldots, Y_{n}\right\}$ veri kümesi yardımıyla $\lambda$ parametresi en çok olabilirlik tahmin yöntemi ile tahmin edilebilir. Burada $Y_{1}$ rasgele değişkeni $\lambda$ parametresi ile üstel dağılıma sahip iken, $Y_{2}, \ldots, Y_{n}$ rastgele değişkenleri, Laplace-Stieltjes dönüşümü (11) ile verilen $F$ dağılım fonksiyonuna sahiptir. $F$ dağ 1 lım fonksiyonunun karakteristik fonksiyonu $\varphi(t)=\left\{1-\frac{i t}{\lambda} e^{L(\lambda-i t)}\right\}^{-1}$ olup ters dönüşüm yöntemi ile $F$ analitik olarak elde edilememektedir. Burada $i=$ $\sqrt{-1}$ 'dir. Bu durumda bu fonksiyonun sayısal olarak hesap edilmesi gerekmektedir. [4] tarafından, karakteristik fonksiyonu bilinen bir rasgele değişkenin dağılım fonksiyonunun sayısal olarak hesap edilebilmesi için aşağıda tarif edilen bir yöntem verilmiştir. $\varphi$ karakteristik fonksiyonuna sahip, ortalaması $\mu$ ve standart sapması $\sigma$ olan bir $F$ dağılım fonksiyonu için

$F(t) \approx \frac{\rho t}{\pi}+\frac{2}{\pi} \sum_{i=1}^{N} \frac{\sin (\rho k t)}{k} C\left(\frac{k}{N}\right) \operatorname{Re} \varphi(\rho k)$

dir. Burada $|t|<1$ için $C(t)=(1-t) \cos \pi t+\frac{1}{\pi}|\sin \pi t|$ ve $|t| \geq 1$ için $C(t)=0, \rho=$ $\frac{\pi}{\mu+3 \sigma+2 t}$ ve $N$ yeterince büyük bir sayıdır [4]. $Y$ rasgele değişkeninin dağılım fonksiyonu (19) denklemi yardımıyla sayısal olarak hesaplanmış ve parametreleri $\alpha=\frac{e^{-\lambda L}\left(e^{\lambda L}-L \lambda\right)^{2}}{e^{\lambda L}-2 L \lambda}$ ve $\beta=\frac{e^{\lambda L}\left(e^{\lambda L}-2 L \lambda\right)}{\lambda\left(e^{\lambda L}-L \lambda\right)}$ olan $L$ ötelemeli gamma dağılımına oldukça yakın çıktı $\breve{g}_{1}$ hesaplamalar sonucunda görülmüştür. Burada $\alpha$ ve $\beta$, (11) denkleminden elde edilen $E(Y)=\frac{e^{\lambda L}}{\lambda}$ ve $E\left(Y^{2}\right)=\frac{2 e^{2 \lambda L}}{\lambda^{2}}-\frac{2 L e^{\lambda L}}{\lambda}$ ifadelerinin $L$ kadar ötelenmiş $\alpha$ ve $\beta$ parametreli gamma dağılımının ilk iki momentine sırasıyla eşitlenmesi ile bulunmuştur. Bu nedenle $Y_{2}, \ldots, Y_{n}$ rasgele değişkenleri için $\alpha$ ve $\beta$ parametreli $L$ ötelemeli gamma dağılımı önerilmiştir. Böylece $Y_{1}$ rasgele değişkeni $\lambda$ parametresi ile üstel dağılıma ve $Y_{2}, \ldots, Y_{n}$ rasgele değişkenleri $\alpha$ ve $\beta$ parametreli $L$ ötelemeli gamma dağılımına sahip olmak üzere $\left\{Y_{1}, Y_{2}, \ldots, Y_{n}\right\}$ veri kümesine dayalı olarak log-olabilirlik fonksiyonu aşağıdaki gibi oluşturulur.

$$
\begin{aligned}
\ln (\ell(\lambda))=-\ln (\lambda) & -\frac{y_{1}}{\lambda}-(n-1) \ln \left(\Gamma\left(\frac{e^{-\lambda L}\left(e^{\lambda L}-L \lambda\right)^{2}}{e^{\lambda L}-2 L \lambda}\right)\right) \\
& -\left(\frac{e^{-\lambda L}\left(e^{\lambda L}-L \lambda\right)^{2}}{e^{\lambda L}-2 L \lambda}\right)(n-1) \ln \left(\frac{e^{\lambda L}\left(e^{\lambda L}-2 L \lambda\right)}{\lambda\left(e^{\lambda L}-L \lambda\right)}\right)-\left(\frac{\lambda\left(e^{\lambda L}-L \lambda\right)}{e^{\lambda L}\left(e^{\lambda L}-2 L \lambda\right)}\right) \sum_{i=2}^{n}\left(y_{i}-L\right) \\
& +\left(\frac{e^{-\lambda L}\left(e^{\lambda L}-L \lambda\right)^{2}}{e^{\lambda L}-2 L \lambda}-1\right) \sum_{i=2}^{n} \ln \left(y_{i}-L\right)
\end{aligned}
$$


burada $\Gamma($.$) gamma fonksiyonudur. Log-olabilirlik fonksiyonu yardımıyla \lambda$ parametresinin en çok olabilirlik $\hat{\lambda}$ tahmin değeri sayısal olarak bulunabilir. Sonuç olarak her sabit $t \geq 0$ için, $\hat{\lambda}$ tahmin değeri ile (17) ve (18) ifadelerinden $M_{d}(t)$ ve $V_{d}(t)$ 'nın tahminlerine ulaşılmış olur.

Not edelim ki ele aldığımız tahmin probleminde bilinen $L$ sabiti bilinmeyen bir parametre olarak gözönüne alınırsa $L$ 'nin tip II sayaç sürecinden gelen bir veri kümesine dayalı olarak $\lambda$ ile birlikte tutarlı bir tahmin edicisi elde edilebilir. Yine bu tutarlı tahmin edicilerin sırasıyla (12) ve (14) ifadelerinde yerine konulmasıyla her sabit $t \geq 0$ için $\widehat{M}_{d}(t)$ ve $\widehat{V}_{d}(t)$ tutarlı tahmin edicileri bulunur. Birinci örneklem planında süreç bağımsız olarak $m$-kez ilk iki olay gerçekleşinceye kadar gözlenirse $m$ tane ilk olayın gerçekleşme zamanlarının aritmetik ortalaması $\lambda$ için tutarlı bir tahmin edici olurken geriye kalan $m$ tane ikinci olay için gerçekleşme zamanlarının minimumu $L$ için tutarlı bir tahmin edici olur. İkinci örnekleme planında süreci bir kez ardışık $n$ olay gerçekleşinceye kadar gözlersek bu durumda (20) de verilen log-olabilirlik fonksiyonu $\lambda$ ve $L$ 'ye bağlı olacaktır. $L$ 'nin tutarlı bir tahmin edicisi olarak veri kümesindeki ilk gözlem hariç geri kalan diğer gözlemlerin minimumu alınır ve $L$ 'nin bu tahmin değerinin olabilirlik denkleminde kullanılması ile $\lambda$ 'nın tutarlı tahmini sayısal olarak elde edilir.

\section{Simülasyon Çalışması}

$\mathrm{Bu}$ bölümde $M_{d}(t)$ ve $V_{d}(t)$ fonksiyonları için önerilen tahmin edicilerinin küçük örneklem hacimlerindeki performanslarının değerlendirilmesi amacıyla bir simülasyon çalışması yapılır. Bu simülasyon çalışması MATLAB paket programı kullanılarak gerçekleştirilmiştir. Tüm simülasyonlar 1000 tekrar üzerine kuruludur ve örneklem hacimleri 10, 30 ve 50 olarak alınmıştır.

Belli bir radyoaktif maddenin $\lambda=2$ oranlı bir $\left\{N_{1}(t), t \geq 0\right\}$ Poisson sürecine göre parçacıklar yaydığını kabul edelim. Sabit bekleme zamanı $L=2$ ve zaman noktaları $t=0.1,0.5,1,3,5$ ve 8 olarak alınsın. Tip II sayaç sürecinin ortalama değer ve varyans fonksiyonları sirasıyla (12) ve (14) denklemlerinden, tahmin değerleri ise (17) ve (18) denklemlerinden hesap edilir. $\mathrm{Bu}$ fonksiyonların gerçek değerleri $M_{d}(t)$ ve $V_{d}(t)$, tahmin edicileri ise $\widehat{M}_{d}(t)$ ve $\widehat{V}_{d}(t)$ olarak aşağıdaki tablolarda verilmiştir. Tablo 1 , birinci örnekleme planı altında elde edilen tahmin edicileri gösterirken Tablo 2, ikinci örnekleme planı altında elde edilen tahmin edicileri göstermektedir. Ayrıca tahmin edicilere ilişkin hata kareler ortalaması (HKO) ve yan değerleri de tablolarda verilmektedir.

Tablo 1. Plan 1 için $\lambda=2$ ve $L=2$ iken Tip II Sayaç Sürecinin $M_{d}(t)$ Fonksiyonu ve Tahmini

\begin{tabular}{|c|c|c|c|c|c|c|c|c|c|c|}
\hline \multirow[b]{2}{*}{$t$} & \multirow[b]{2}{*}{$M_{d}(t)$} & \multicolumn{3}{|c|}{$n=10$} & \multicolumn{3}{|c|}{$n=30$} & \multicolumn{3}{|c|}{$n=50$} \\
\hline & & $\widehat{M}_{d}(t)$ & $\begin{array}{l}\boldsymbol{H K O} \\
\left(\widehat{\boldsymbol{M}}_{\boldsymbol{d}}(\boldsymbol{t})\right)\end{array}$ & $\begin{array}{c}\text { Yan } \\
\left(\widehat{M}_{d}(t)\right)\end{array}$ & $\widehat{M}_{d}(t)$ & $\begin{array}{c}\text { HKO } \\
\left(\widehat{M}_{\boldsymbol{d}}(\boldsymbol{t})\right)\end{array}$ & $\begin{array}{c}\text { Yan } \\
\left(\widehat{M}_{d}(t)\right)\end{array}$ & $\widehat{M}_{d}(t)$ & $\begin{array}{c}H K O \\
\left(\widehat{M}_{d}(t)\right)\end{array}$ & $\begin{array}{c}\text { Yan } \\
\left(\widehat{M}_{d}(t)\right)\end{array}$ \\
\hline 0.1 & 0.1813 & 0.1820 & 0.0026 & 0.0007 & 0.1807 & 0.0009 & -0.0006 & 0.1808 & 0.0006 & -0.0005 \\
\hline 0.5 & 0.6321 & 0.6117 & 0.0078 & -0.0204 & 0.6266 & 0.0017 & -0.0055 & 0.6267 & 0.0008 & -0.0054 \\
\hline 1 & 0.8647 & 0.8391 & 0.0084 & -0.0256 & 0.8554 & 0.0025 & -0.0093 & 0.8600 & 0.0015 & -0.0047 \\
\hline 3 & 1.0183 & 1.0155 & 0.0001 & -0.0028 & 1.0178 & 0.0000 & -0.0005 & 1.0181 & 0.0000 & -0.0002 \\
\hline 5 & 1.0916 & 1.1133 & 0.0057 & 0.0217 & 1.1009 & 0.0023 & 0.0093 & 1.0946 & 0.0013 & 0.003 \\
\hline 8 & 1.2015 & 1.2653 & 0.0375 & 0.0638 & 1.2192 & 0.0114 & 0.0177 & 1.2161 & 0.0077 & 0.0146 \\
\hline
\end{tabular}


Tablo 2. Plan 1 için $\lambda=2$ ve $L=2$ iken Tip II Sayaç Sürecinin $V_{d}(t)$ Fonksiyonu ve Tahmini

\begin{tabular}{|c|c|c|c|c|c|c|c|c|c|c|}
\hline \multirow[b]{2}{*}{$t$} & \multirow[b]{2}{*}{$V_{d}(t)$} & \multicolumn{3}{|c|}{$n=10$} & \multicolumn{3}{|c|}{$n=30$} & \multicolumn{3}{|c|}{$n=50$} \\
\hline & & $\widehat{V}_{d}(t)$ & $\begin{array}{c}\text { HKO } \\
\left(\widehat{\boldsymbol{V}}_{\boldsymbol{d}}(\boldsymbol{t})\right)\end{array}$ & $\begin{array}{c}\text { Yan } \\
\left(\widehat{V}_{d}(t)\right)\end{array}$ & $\widehat{V}_{d}(t)$ & $\begin{array}{c}\boldsymbol{H K O} \\
\left(\widehat{\boldsymbol{V}}_{\boldsymbol{d}}(\boldsymbol{t})\right)\end{array}$ & $\begin{array}{c}\text { Yan } \\
\left(\widehat{V}_{d}(t)\right)\end{array}$ & $\widehat{V}_{d}(t)$ & $\begin{array}{c}\text { HKO } \\
\left(\widehat{V}_{\boldsymbol{d}}(t)\right)\end{array}$ & $\begin{array}{c}\text { Yan } \\
\left(\widehat{V}_{d}(t)\right)\end{array}$ \\
\hline 0.1 & 0.1484 & 0.1455 & 0.0008 & -0.0029 & 0.1467 & 0.0003 & -0.0017 & 0.1468 & 0.0002 & -0.0016 \\
\hline 0.5 & 0.2325 & 0.2250 & 0.0008 & -0.0075 & 0.2294 & 0.0003 & -0.0031 & 0.2312 & 0.0002 & -0.0013 \\
\hline 1 & 0.1170 & 0.1254 & 0.0030 & 0.0084 & 0.1201 & 0.0012 & 0.0031 & 0.1207 & 0.0007 & 0.0037 \\
\hline 3 & 0.0229 & 0.0340 & 0.0010 & 0.0111 & 0.0259 & 0.0002 & 0.003 & 0.0249 & 0.0001 & 0.002 \\
\hline 5 & 0.0839 & 0.0998 & 0.0037 & 0.0159 & 0.0896 & 0.0014 & 0.0057 & 0.0879 & 0.0009 & 0.004 \\
\hline 8 & 0.1777 & 0.2016 & 0.0132 & 0.0239 & 0.1882 & 0.0055 & 0.0105 & 0.1829 & 0.0033 & 0.0052 \\
\hline
\end{tabular}

Tablo 3. Plan 2 için $\lambda=2$ ve $L=2$ iken Tip II Sayaç Sürecinin $M_{d}(t)$ Fonksiyonu ve Tahmini

\begin{tabular}{|c|c|c|c|c|c|c|c|c|c|c|}
\hline \multirow[b]{2}{*}{$t$} & \multirow[b]{2}{*}{$M_{d}(t)$} & \multicolumn{3}{|c|}{$n=10$} & \multicolumn{3}{|c|}{$n=30$} & \multicolumn{3}{|c|}{$n=50$} \\
\hline & & $\widehat{M}_{d}(t)$ & $\begin{array}{c}\text { HKO } \\
\left(\widehat{M}_{d}(t)\right)\end{array}$ & $\begin{array}{c}\text { Yan } \\
\left(\widehat{M}_{d}(t)\right)\end{array}$ & $\widehat{M}_{d}(t)$ & $\begin{array}{c}\text { HKO } \\
\left(\widehat{M}_{d}(t)\right)\end{array}$ & $\begin{array}{c}\text { Yan } \\
\left(\widehat{M}_{d}(t)\right)\end{array}$ & $\widehat{M}_{d}(t)$ & $\begin{array}{c}\text { HKO } \\
\left(\widehat{M}_{d}(t)\right)\end{array}$ & $\begin{array}{c}\text { Yan } \\
\left(\widehat{M}_{d}(t)\right)\end{array}$ \\
\hline 0.1 & 0.1813 & 0.1978 & 0.0007 & 0.0165 & 0.1896 & 0.0007 & 0.0083 & 0.1855 & 0.0006 & 0.0042 \\
\hline 0.5 & 0.6321 & 0.6819 & 0.0134 & 0.0498 & 0.6159 & 0.0093 & -0.0162 & 0.6320 & 0.0010 & -0.0001 \\
\hline 1 & 0.8647 & 0.8739 & 0.0155 & 0.0092 & 0.8736 & 0.0099 & 0.0089 & 0.8701 & 0.0022 & 0.0054 \\
\hline 3 & 1.0183 & 0.9689 & 0.0009 & -0.0494 & 1.0246 & 0.0009 & 0.0063 & 1.0199 & 0.0002 & 0.0016 \\
\hline 5 & 1.0916 & 1.0768 & 0.0074 & -0.0148 & 1.0799 & 0.0060 & -0.0117 & 1.0899 & 0.0013 & -0.0017 \\
\hline 8 & 1.2015 & 1.1732 & 0.0298 & -0.0283 & 1.1941 & 0.0091 & -0.0074 & 1.1992 & 0.0067 & -0.0023 \\
\hline
\end{tabular}

Tablo 4. Plan 2 için $\lambda=2$ ve $L=2$ iken Tip II Sayaç Sürecinin $V_{d}(t)$ Fonksiyonu ve Tahmini

\begin{tabular}{|c|c|c|c|c|c|c|c|c|c|c|}
\hline \multirow[b]{2}{*}{$t$} & \multirow[b]{2}{*}{$V_{d}(t)$} & \multicolumn{3}{|c|}{$n=10$} & \multicolumn{3}{|c|}{$n=30$} & \multicolumn{3}{|c|}{$n=\mathbf{5 0}$} \\
\hline & & $\widehat{V}_{d}(t)$ & $\begin{array}{c}H K O \\
\left(\widehat{V}_{d}(t)\right)\end{array}$ & $\begin{array}{c}\text { Yan } \\
\left(\widehat{V}_{d}(t)\right)\end{array}$ & $\widehat{V}_{d}(t)$ & $\begin{array}{c}\text { HKO } \\
\left(\widehat{\boldsymbol{V}}_{\boldsymbol{d}}(\boldsymbol{t})\right)\end{array}$ & $\begin{array}{c}\text { Yan } \\
\left(\widehat{V}_{d}(t)\right)\end{array}$ & $\widehat{V}_{d}(t)$ & $\begin{array}{c}H K O \\
\left(\widehat{V}_{d}(t)\right)\end{array}$ & $\begin{array}{c}\text { Yan } \\
\left(\widehat{V}_{d}(t)\right)\end{array}$ \\
\hline 0.1 & 0.1484 & 0.1582 & 0.0003 & 0.0098 & 0.1428 & 0.0002 & -0.0056 & 0.1455 & 0.0002 & -0.0029 \\
\hline 0.5 & 0.2325 & 0.2160 & 0.0104 & -0.0165 & 0.2366 & 0.0072 & 0.0041 & 0.2320 & 0.0007 & -0.0005 \\
\hline 1 & 0.1170 & 0.1007 & 0.0113 & -0.0163 & 0.1082 & 0.0111 & -0.0088 & 0.1102 & 0.0083 & -0.0068 \\
\hline 3 & 0.0229 & 0.0293 & 0.0008 & 0.0064 & 0.0276 & 0.0004 & 0.0047 & 0.0230 & 0.0002 & 0.0001 \\
\hline 5 & 0.0839 & 0.0712 & 0.0113 & -0.0127 & 0.0740 & 0.0062 & -0.0099 & 0.0801 & 0.0012 & -0.0038 \\
\hline 8 & 0.1777 & 0.1556 & 0.0235 & -0.0221 & 0.1720 & 0.0079 & -0.0057 & 0.1828 & 0.0031 & 0.0051 \\
\hline
\end{tabular}

$t$ zaman noktası, $L$ sabit bekleme zamanı değerine yaklaştıkça tahmin edicilere ilişkin HKO değerleri her iki örnekleme planı altında da artmaktadır. HKO değerlerindeki bu artışın, Plan 2'den elde edilen tahmin edicilerde daha fazla olduğu görülmektedir. $t$ zaman noktasının, $L$ sabit bekleme zamanı değerine yakın olan değerleri için Plan 2 altında elde edilen tahmin edicilere ait HKO değerlerinin, Plan 1'e göre elde edilen tahmin edicilerin HKO değerlerinden daha fazla olduğu tablolardan söylenebilir. Ayrıca büyük $t$ değerleri için Plan 1'e göre elde edilen tahmin edicilerin HKO değerleri, Plan 2 altında elde edilen tahmin edicilerin HKO değerlerine göre daha hızlı artmaktadır.

Her bir örnekleme planı için örneklem hacminin artmasıyla birlikte tahmin edicilere ait HKO değerlerinin de azaldığı görülmektedir. Örneğin, $t$ zaman noktası 1 olarak seçildiğinde, varyans fonksiyonunun Plan 1'e göre elde edilen tahmin edicisine ilişsin HKO değerleri, $n=10$ için $0.0030, n=30$ için 0.0012 ve $n=50$ için 0.0007 olarak elde edilmiştir. Açıktır ki, örneklem hacminin artmasıyla HKO değerleri azalmakta ve tahmin edicilerin performansları artmaktadır. Benzer durum Plan 2 için de geçerlidir.

\section{Sonuç}

Bu çalışmada tip II sayaç sürecinin varyans fonksiyonu analitik olarak elde edilmiştir. Daha sonra bu sürecin ortalama değer ve varyans fonksiyonları için uygulamada karşılaşılabilecek örneklem planları dikkate alınarak birer parametrik tahmin edicileri önerilmiş ve tutarlı oldukları gösterilmiştir. Önerilen tahmin edicilerin değerlendirilmesi amacıyla bir simülasyon çalışması yapılmıştır. Yapılan simülasyon çalışması ile bu tahmin edicilerin küçük örneklem için bile iyi bir performans sergiledikleri ortaya çıkmıştır. 


\section{Kaynakça}

[1] E. W. Frees, "Warranty analysis and renewal function estimation," Nav. Res. Logist. Q., vol. 33, pp. 361-372, 1986a.

[2] E. W. Frees, "Nonparametric Renewal Function Estimation," Ann. Statist., vol. 14, no. 4, pp. $1366-$ $1378,1986 \mathrm{~b}$.

[3] H. Aydoğdu and F. Öztürk, "Estimation of the variance function in renewal processes," Journal of Applied Statistical Science, vol.7, no. 4, pp. 191-202, 1999.

[4] H. Bohman, "A method to calculate the distribution function when the characteristic function is known," BIT, vol.10, no. 3, pp. 237-242, 1970.

[5] S. Karlin and H. M. Taylor, A First Course in Stochastic Processes, $2^{\text {nd }}$ Edition. New York: Academic Press, 1975.

[6] W. L. Smith, "Renewal theory and its ramifications," J. Roy. Stat. Soc. B., vol. 20, pp. 243-302, 1958.

[7] G. R. Grimmett and D. R. Stirzaker, Probability and Random Processes. New York:Oxford University Press Inc., 1992.

[8] S. Biswas and H. D. Arora, "On an application of Geiger-Muller counter model (Type-II) for optimization relating to hospital administration," Acta Med. Int., vol. 4, pp. 16-19, 2017.

[9] L. Pal and I. Pazsit, "On some problems in the counting statistics of nuclear particles investigation of the dead time problems," Nucl. Instrum. Methods Phys. Res. A, vol. 693, pp. 26-50, 2012.

[10] R. P. Gardner and L. Lianyan, "On extending the accurate and useful counting rate range of GM counter detector systems," Appl. Radiat. Isot. Vol. 48, no. 10--12, pp. 1605-1615, 1997. 